\title{
Demokratiekrise zwischen Ungleichheit und Wohlfahrtsstaat
}

\section{DR. KONRAD HUMMEL}

ist Diplompädagoge und Sozialwissenschaftler und war von 2002 bis 2008 Sozialdezernent der Stadt Augsburg, danach unter anderem Abteilungsleiter Politik beim Bundesverband für Wohnen und Stadtentwicklung e. V. (vhw) in Berlin, Beauftragter des Oberbürgermeisters für die Konversion in Mannheim sowie Geschäftsführer der Mannheimer Wohn- und Stadtentwicklungsgesellschaft MWSP.

\author{
Die Demokratie in Deutschland beinhaltet ein \\ Sozialstaatsgebot, das soziale Gerechtigkeit mehren \\ und Differenzen im "sozialen und demokratischen \\ Bundesstaat"(Art. 20 Grundgesetz) mindern will. \\ Dementsprechend gilt umgekehrt, dass die gefühlte \\ Infragestellung von Gerechtigkeit und Sicherheit auch \\ an den Wurzeln der Demokratie nagt. Dies ist eines \\ der Merkmale der derzeitigen Demokratiekrise.
}

Da Gerechtigkeit und soziale Sicherheit schwer messbar und objektivierbar sind, geht es darum, die geschichtliche Aufladung dieser Ziele und ihre Wahrnehmung genauer zu verstehen. Die Bundesregierung geht in Teilen von der gleichen Analyse aus und hat "ungleiche Lebensverhältnisse « zu einem grundsätzlichen Politikfeld erklärt, für das eine Kommission im Sommer 2019 Empfehlungen ausarbeiten soll.

Im Mittelpunkt der Debatte um diese Kommission stehen der Stadt-Land-Unterschied, Verschuldung und Infrastruktur sowie Arbeitsgruppen der bekannten Akteure dazu (z.B. Städtetag etc.). Die hier vorgelegte Analyse folgt diesen Schwerpunkten nicht, sondern stellt die Veränderungen des gesellschaftlichen Zusammenhalts, die Heterogenität der Gesellschaft und die Grenzen des deutschen Sozialstaatsprinzips in einer Europäischen Union in den Mittelpunkt.

\section{Die Repolitisierung des Sozialstaates}

Während der Sozialstaat lange Zeit im "Gewand « sozialer Träger daherkam, haben sich die Bedarfslagen so vervielfältigt und verändert, ist die Dynamik so schnell und die Entscheidungen so mehrdeutig geworden, dass der Staat selbst in den Mittelpunkt der Aufmerksamkeit rückt. Der deutsche Sozialstaat sendet mehrere Botschaften gleichzeitig an eine divers gewordene heterogene Gesellschaft. Sozialgesetze werden immer wieder nach- gebessert, neue Rechtslagen aufgemacht, das Wohlfahrtswesen ideell hochgehalten. Andererseits wird ständig gekürzt, rationalisiert, Marktkonkurrenz aufgemacht und Missbrauchsvermeidung perfektioniert. Der Sozialstaat ist zur Dauerbaustelle geworden, dessen Infrastruktur keiner Systematik mehr folgt, was die Kommunen auf den Plan gerufen hat. Sie treiben(müssen) die Sozialstaatslage vorantreiben z.B. durch Beratungsstellen gegen Diskriminierung, für Behinderte, neue Wohnformen, Unterkünfte, Quartiersmanager, Seniorentreffpunkte usw. vor allem wenn sich »katholische und evangelische Träger « mal wieder nicht einigen konnten, Neutralität gefragt ist oder verdeckte Subventionierungen notwendig sind (billige Grundstücke etc.).

$\mathrm{Zu}$ einem wichtigen Indikator des Sozialstaates ist geworden, wohin die Aufmerksamkeit geht, welche Zielgruppe im Mittelpunkt steht, welche Not als »neue soziale Frage « jeweils definiert wird (derzeit die Großstadtmieten). Dieses Jahrzehnt 2010-2019 war besonders von Zuwanderung, Migration, Asyl, Flüchtlingen und den Langzeitfolgen bei unterschiedlich ethnisch-religiösen Gruppen geprägt. Entsprechend dem versteckten »Leitbild « deutscher Sozialstaatlichkeit stand dabei die Versorgungs- und Fürsorgelage für Flüchtlinge im Mittelpunkt, nicht deren Rechtslagen, Bürger- und Wahlrechte. Dies verstärkte Gefühle der Ungleichheit, weil " einheimische « soziale Gruppen am Rande von Prekariat und Arbeitsplatzsicherheit 
sich massiv benachteiligt fühlten (nicht mehr wahrgenommen) und den Sozialstaat völlig ungerecht empfinden. »Wir sind das Volk« ist eine Kampfformel, die das Sozialstaatsprinzip weit vor das Grundgesetz verschiebt hin zu ethnischer Zugehörigkeit und das Gemeinwesen dramatisch aufspaltet in Gruppen, die mehr und die weniger Anrechte auf Sozialleistungen haben sollen. Latent sind solche Konflikte auch zwischen den Generationen, den unterschiedlichen Lebensstilen (Ehe für alle) und Betreuungsformen (Zahlungen für Kinder im Kindergarten oder in der Familie) angelegt. dass das Vertrauen wächst, dass es kompetente Menschen (Instanzen/Indikatoren) gibt, die genauer wissen als der "gesunde Menschenverstand ", welche Symptome wirklich langfristige Brüche verursachen?

Geht es am Ende nicht viel mehr um Demokratie als um "Sozialstaat « im Sinne alter patriarchaler Fürsorge, weil es bei Demokratie immer auch um Mitverantwortung geht und bei Fürsorge immer auch um Erwartung, dass Andere meine Probleme lösen? Und geht es am Ende nicht mehr um die Rahmenbedingungen, unter denen etwas wahrgenommen, verfestigt

\section{"Der Sozialstaat ist zur Dauerbaustelle geworden, dessen Infrastruktur keiner Systematik mehr folgt."}

Die Konflikte werden nicht mehr im Betrieb ausgetragen (»Klassenkampf «) oder zwischen weltanschaulichen Gruppen (wie noch beim $\$ 175$ zur Homosexualität), sondern über den Staat. Der hat in Deutschland Fürsorge versprochen, der hat Wohlfahrtsverbände beauftragt, der soll nun die richtigen Prioritäten setzen. Sogar die Wohlfahrtsverbände "zerdrückt es fast in diesen Auseinandersetzungen, weil ihr ethisches Gebot Solidarität und Professionalität verlangt, große Teile ihrer Klientel dies aber weder wahrnehmen noch ohne weiteres akzeptieren will und der Staat für Professionalität nicht immer die angemessene garantieren kann. Die politischen Parteien nehmen in der ausklingenden Merkel-Ära diese Verschiebung zum Staat an, indem sie zahllose Einzelgesetze und Leistungen nachbessern.

Nun soll es bei "gleichen Lebenschancen « der Kommission der Regierung erneut um materielle Nachbesserungen gehen, auf dem Land, bei verschuldeten Gemeinden, in der Infrastruktur- in der Annahme, die Bevölkerung gewinnt wieder Vertrauen in die Demokratie, weil sie weiß das es "allen gleich gut oder gleich schlecht " geht. Ist dies eine realistische Erwartung auf Seiten der Bevölkerung wie auf Seiten des Staates in einer gesellschaftlichen Phase, die durch extreme Dynamik, Vervielfältigung und Unterschiede geprägt ist?

Muss es nicht viel mehr darum gehen, dass das Vertrauen wächst in die Instrumente des Staates, dass diese angemessen und erfolgreich zum Einsatz kommen, und vermeintlich gelöst wird - also die Bedingungen eher zu verändern als individuelle Lagen und Meinungen!? So haben wir im Bildungsaufbruch der siebziger Jahre wahrgenommen, dass eine gut ausgebildete Republik auch Probleme anders wahrnimmt und anpackt. So müssen wir es noch lernen bei »territorialer Ungleichheit«, die unsere Sicht auf die Dinge mehr prägt als uns hilfreich sein kann ( was uns immer noch im Zuge der Ost-West-Vereinigung beschäftigt, längst aber in den Städten und Quartieren Folgen hat).

Dass es derart "ungleiche Lebensorte « gibt, dient politisch Vielen als ständiger Quell der Wut, der Angst, der Abgrenzung und der Beweisführung gegenüber den unfähigen Eliten des Staates. Die mediale No-go-area-Berichterstattung bedient diese tiefe Quelle, mit der auch leicht nachzuvollziehen ist, warum in Städten fernab mit weniger Migranten mehr Ausländerfeindlichkeit und Staatsverdrossenheit vorherrscht als in Städten mit vielen Migranten, weil eine Art »Sodom und Gomorrah «Effekt einsetzt(»sieh dich nicht um, es könnte dich erstarren lassen «). Soziale Unterschiede, mit denen sich Deutschland schwerer tut als z. B Frankreich oder - noch mehr - die USA. An vielen diesen Orten stehen die Gegensätze in einem inneren Spannungsfeld zueinander. So können sich Land und Stadt auch ergänzen(was lange Zeit so im zentralistischen Frankreich war), gegenspiegeln; Arm und Reich sich architektonisch hochschaukeln (New York) oder unterschiedliche Lebensstile markieren (Nord/Süditalien). Ab wann sind Gegensätze Spaltpilze demokratischen Vertrauens? Sind sie rein empirisch an Lebensdaten messbar?

\section{Das ungeklärte Gleichgewicht von Sozialstaatsprinzip, Demokratie und Gesellschaftspolitik}

Die letzten Jahre haben zur Krise der Demokratie eine umfangreiche Literatur und Beschäftigung mit der Demokratie selbst hervorgerufen. Dabei wird der rein wissenschaftliche Rahmen schnell gesprengt - wie er z.B. mit der ersten Großstadtstudie zur lokalen Demokratie noch publiziert worden war (van Deth 2014). Bis dahin wurde das Paradoxon beschworen, die überwiegende Mehrzahl der Menschen seien sehr zufrieden mit der Demokratie im Land, aber im Detail dann doch nicht berechenbar, vom Wutbürger über die »ungeladenen Aussenseiter« (siehe van Deth 2014, S. 167) bis zu den auffälligen Abweichungen der Einstellungen in unterschiedlichen Wohnbezirken.

Hellhörig hätte damals schon machen müssen, dass sich in bestimmten Quartieren innere und in bestimmten ganz anderen Wohnlagen äußere Abwendungen von der Demokratie verfestigt haben, die mit Sachfragen nichts zu tun haben. Quartiere manifestieren Bruchstellen, die Menschen dort ziehen sich zurück (Wahl»verweigerung« bei gleichzeitig hohem staatlichen Transfereinsatz) oder sie greifen das Angebot von menschenverachtenden Protestparteien auf, die damit durchaus zur relevanten Größe demokratischer Willensbildung werden. Während die einen nicht mehr zur Wahl gehen, doppelt so viele von ihnen (im Unterschied zu befragten Bürgern anderswo) kein Vertrauen in Politik haben und auch doppelt so viele über keine Nachbarschaftskontakte verfügen(Quelle: s.o.), wenden die anderen in sehr homogenen Quartieren sich ab. Sie fühlen sich zu wenig respektiert $(40 \%$ zu 10\% bei anderen Gruppen), halten die Migrantenfrage für die zentrale Frage der Politik (während weltoffene Bürger die Klimapolitik dort sehen) und wenden sich rechtsnationalen Politikangeboten zu (Faus/Storks 2019). Natürlich ist nicht die Wohnlage, das Quartier, die eigentliche Ursache der Einstellungen und Probleme. Die Quartiere und ihre »Gegenquartiere " bestätigen jeden Tag die Einstellungen, geben den Resonanzboden und sind Sym- 
ptom für Bruchstellen der Demokratie und des Vertrauens in den Sozialstaat.

Die in den letzten 20 Jahren zunehmend stark segregierten Wohnquartiere in der Republik sind zum Echoraum (hier tönt das Vorurteil zurück) geworden, sie bestärken eine Infragestellung des sozialstaatlichen Ordnungsgefüges: Es funktioniert so nicht mehr.

\section{Die gesellschaftlichen Ränder bekommen ihre politische Stimme}

Wer sich mit Ungleichheit und Zusammenhalt in der Gesellschaft beschäftigt, muss sich mit diesen territorialen Ungleichheiten und den damit einhergehenden Verstärker/ Echoeffekten auseinandersetzen, bei denen sich die Gleichen bestätigt sehen, teil von Fakten gestützt, teils unabhängig von der Faktenlage. In einer sich in Milieus, Gruppen und Heterogenitäten entfaltenden Gesellschaft ist die Einstellung zu Staat und Sozialstaat auch ein »Gruppenmerkmal«, es markiert Gemeinsamkeit meiner Gruppe gegenüber allen Anderen. Die demokratieund sozialstaatskritische Einstellung ist selbst ein Identitätsfaktor dieser Gruppen.

Während die meisten Demokratiepublikationen der letzten Jahre allgemeiner sich am Niveau der Weltoffenheit oder Fremdenfeindlichkeit, letztlich der Integration der Republik, abarbeiteten, widmeten sich nur einige dem wirklichen Verstehen der Ausgegrenzten (typisch dafür vielleicht die Diakoniekampagne: Die Unerhörten). Aus USA und Frankreich kamen beindruckende Literaturbeispiele über die abgehängten (Trump)wähler oder die Verbitterten des französischen Nordostens. Die Göttinger Forschungsgruppe um Walter gab nicht so sehr den (mittelständischen) Wutbürgern dafür aber den »Entbehrlichen « (2011) in den alten Arbeiterquartieren eine Stimme. Und für eine »dritte Generation « von $\mathrm{Zu}$ wanderern gilt, dass sie Demokratie transnational in Frage stellen. Sie reagieren auf »ihre Heimatpolitik von Erdogan « und bringen sie gegen die deutsche Demokratie in Stellung.

Die einfachen Antworten (Demokratie heißt Regierung durch das Volk und Mehrheit zählt) reichen deshalb nicht mehr. Die Krise der Demokratie ist nicht ihr Parteienstreit, sondern der Mangel an Identifikation mit dem Ordnungs- und Sozialstaatsgefüge. Es ist davon auszugehen, dass ein Drittel der Bevölkerung solche Zweifel hat. Die Erosion geht schleichend und betrifft nicht nur migrantische Teilgruppen und sozial abgehängte Deutsche, sondern auch Steuer- und Gemeinschaftsflüchter, Menschen, die sich aus staatlichen Strukturen raushalten, nicht zuletzt ihre Kinder aus Problemgebieten abmelden. Für alle gilt, dass sie dem Sozialstaatsversprechen nicht mehr glauben, dass es allen besser gehen kann.

Die Integrationspolitik und die Flüchtlingsfrage haben vermutlich die notwendigen Positionsklärungen nur beschleunigt und forciert, weshalb die Ebertstiftung gleich auf der einen Skala die »national orientierten " und am anderen Ende die »weltoffenen « konstruiert (statistisch interessanterweise ähnlich groß!) Dazwischen die verunsicherte Mitte, aus der heraus ständig "Ausreißversuche« erfolgen wie die Gelbwesten Frankreichs. Wie fragil das alles geworden ist zeigt, dass solche "Ausreißversuche" derzeit durch nichts und niemand »repräsentativ« aufgefangen werden und hoffnungslos verbittert, hassgeprägt und antistaatlich enden.

Der französische Geograph Christophe Guilluy (FAZ,24.11.18) nimmt zu den Gelbwesten das Bild der Grenzen auf und sagt: Das Geld bildet die Grenze, der gegenüber der Kleinbürger machtlos ist, wenn sein Krankenhaus vor Ort schließt, der reichere Bürger umziehen kann. Wenn viele Bürger nationale Grenzen wollen, ist es letztlich der Ruf nach dem Staat, der sie schützt gegen den gesamten Wandel, ob er offen kommt (als Flüchtling) oder versteckt (über Digitalisierung, Effizienz, Arbeitsplatzverlagerung). auf, S.19,19.2.19/FAZ vom 6.5.2019, S. 6: Schuld und Sühne).

Während viele Jahre in Deutschland im Sozialstaat alles als »Neiddiskussion« thematisiert wurde (wie gehen wir mit Ungleichheiten um), ist es heute ein Kampf aller gegen alle geworden, weil wir nicht wissen, wie es den Anderen geht und ob "wir« noch wertgeschätzte Mitbürger sind. Gefühlt sind die Gewinner von heute nicht mehr »die Kapitalisten «, sondern die »Bildungsbürger mobiler multikultureller Art«. "Die verhassten Weltbürger" spiegelt die ZEIT Ende 2018 (Nr. 47, S. 49) ihrer Leserschaft die Sicht der »Einfachen « auf die »Gebildeten« - der Widerspruch, der heute stärker Klassen trennt als der Kapitalbesitz. Vorsichtig formuliert ein ökobeseelter Selbstversucher: "Man muss aufpassen, dass man nicht zum Bild einer BionadeBourgoisie beiträgt, die allen kleinen Leuten erklärt, wie es zu gehen hat (FAZ, 12.4.19) Wer über die »Unerhörten« oder »Abgehängten« redet, muss gleichzeitig über die »Besserwisser « und »Nestflüchter « reden, muss sich auf die Suche nach der "Mitte" der Gesellschaft machen.

Allen aber, ob einfach-gebildete, national-mittelständische oder weltoffene Bürge*rinnen ist gemeinsam, dass sie miteinander verbunden sein wollen, dass sie Zusammenhalt suchen! Alle drei Gruppen der Ebert Studie suchen mehrheitlich den gesellschaftlichen Zusammenhalt. Aber jede Gruppe versteht etwas anderes darunter und tut unterschiedliches selbst dafür! In der politischen Diskussion wird

\section{"Die Krise der Demokratie ist nicht ihr Parteienstreit, sondern der Mangel an Identifikation mit dem Ordnungs- und Sozialstaatsgefüge."}

Die Polarisierung bedeutet in der Folge, dass alle sich absetzen in der Annahme, die Anderen würden sie diskriminieren oder bevorzugt werden. Der an- und wahrgenommenen Wut- und Kränkung auf der einen Seite der Gesellschaft steht eine Identitätspolitik intellektueller moderner Gruppen gegenüber, die ständig und überall Diskriminierung, Rassismus und Entwertung ortet und die sozialen Trennungsmauern der Gesellschaft massiv weiter verstärkt (NZZ vom 19.2.2019, S. 19: Das Wir löst sich dieser Punkt von gemeinsamen Vorstellungen, »Versprechungen « der Demokratie zu wenig reflektiert.

Dies ist der Referenzpunkt der Gleichheits-/Ungleichheitsdiskussion in der Demokratie! Wie weit entfernt sich jede Gruppe vom Zusammenhalt der Gesellschaft, wie trägt sie zum Zusammenhalt bei, wie sehr setzt sie sich $a b$ ?

Ausgehend von diesem Koordinatensystem ist der Unterschied Stadt- Land, wie ihn die tagespolitische Debatte der Kommission für gleichwertige Lebensbe- 
dingungen formuliert, sehr relativ. Die Ressourcenausstattung mag sehr verschieden bis ungleich sein ohne den Zusammenhalt und die Akzeptanz der Demokratie zu gefährden. Wenn aber die Einstellungen und das Wertesystem der Menschen nicht mehr nur individuell sondern in großen Gruppen und manifest in Quartieren sich entfernt, treten Spaltungen auf, die die Demokratie nachhaltig schädigen. Eine Spirale von Misstrauen und Verweigerung von Kooperation und demokratischer Mitgestaltung wird in Gang gesetzt. Unterfüttert wird der Prozess, in dem z.B. die Bildungschancen dieser Gruppen nachhaltig schlechter sind und die Perspektiven damit auch langfristig nicht mehr irgendeinem Integrations- und Aufstiegsideal entsprechen. Der französische Präsident Emmanuel Macron spricht mit Blick auf einige Quartieren sogar von "Sezession" und sie weit über eine sozialpolitische Wahrnehmung hinausgreifend staatsrechtliche Verfassungsgrundlagen in Frage gestellt.

Dass es hier um mehr als um sozialstaatliche Leistungen geht und Politikmechanismen zu kurz greifen, analysiert 2018/19 eine Gruppe Kommunalpolitiker im »Appell und Pakt von Mannheim für urbane Demokratie " (siehe dazu BdW 4/2019, S. 132). Die klassischen Fördermechanismen würden Ungleichheit in diesem Sinne verschärfen: Kein weiter so! Es ist bitter und bezeichnend für die deutsche Sozialstaatsentwicklung, dass der aktuelle Quartiersappell aus Mannheim nahtlos anknüpfen muss an Aussagen von 1977 zum »Infrastrukturausgleich als sozialpolitische Aufgabe (Herlyn 1977).

Dort kommt Herlyn aufgrund seiner Studien zum Schluss, dass sich »ein egalitärer Ansatz politischer Strategie verbietet, die Gleichverteilung propagiert, jedoch Ungleichheit schafft «(aaO. S. 586), wenn man bürgernah den Gruppen helfen will, die von Defiziten in der Versorgungssituation geprägt sind. Wenn eine benachteiligte Personengruppe »festhängt « in ihrer Gruppe, an ihrem Arbeitsplatz, in ihrem Quartier, das längst geprägt ist von Problemen und Vielfalt, verschärfen individuelle Förderungen und egalitäre Leistungen die Ungleichheit.

Ein wirksamer Sozialstaat wird oft vor der Entscheidung stehen »egalitär zu liefern " oder "gezielt asymmetrisch « und strukturell territorial zu unterstützen. Es kann für die Klärung von Gleichheit und Zusammenhalt zentral sein, ob es ernsthaft darum geht, Lebenschancen (nach) zu bessern oder Versorgung aufrechtzuer- halten. Im Kern tauchte dies in der HartzIV-Debatte ebenso auf wie es auch Gegenstand offensiver Entwicklungspolitik ist. Und es steckt in jeder Qualitätsdebatte z.B. von Erziehungsdienstleistungen: verwahrt der Kindergarten und stellt die Berufstätigkeit der Mutter sicher oder/und entfaltet er alle Chancen des Kindes. Das ist nicht immer und jederzeit das gleiche! leistungen angemessen sind. Wie also kann "Zusammenhalt " und hinreichende »Gleichheit " gemeinsamer verstanden und transparenter gehandelt werden? In der Zuwanderungsdebatte, der Integrationsfrage dieser Gesellschaft, kann man sich des Eindrucks nicht erwehren, dass jede Gruppe bedient werden soll in einem in sich nirgendwo stringenten Kompromiss:

\section{"Der Zusammenhalt einer Gesellschaft ist zu allererst eine Frage dessen, was uns untereinan- der und miteinander wichtig ist und nicht, was 'der abstrakte Staat ‘ür uns erledigen soll."}

Steht die Demokratie im Mittelpunkt der Bemühungen, muss es um die Chancen gehen, um die »Versprechungen « des »demokratischen und sozialen Bundesstaates« (Art. 20). Versprechungen, die nur mit den Betroffenen eingelöst werden können und nur erfolgreich sind, wenn viele andere Gruppen den Erfolg der bestimmten Gruppen auch wollen, Aufstieg zulassen. Der Sozialstaat ist nicht zu reduzieren auf eine Finanzierungsgröße, er ist ein zentrales Element innerhalb der Demokratie nach 1948. Selbst die Klärung der aktuellen »Respekt «- Rente ist eine Frage, wieviel Wert allen Generationen die Finanztransfers sind für einen Teil der älteren Mitbürger. In einer pluralistischen Gesellschaft wird es notwendiger denn je sein, nicht vermeintlich objektive Lebensstandards, Warenkörbe oder Pflegesatzberechnungen zu haben, sondern die Klärung, wieviel die Verbesserung dieser Lebenslagen uns wert ist, auch wenn sie auf Kosten anderer Maßnahmen/ Zielgruppen geht. Cremer formuliert das kurz (in: Wendt 2014, S. 98.): »Eine Sozialpolitik ist gegen die Mitte der Gesellschaft nicht aufrechtzuerhalten«.

\section{Gesellschaftlicher Zusammenhalt}

Der Zusammenhalt einer Gesellschaft ist $\mathrm{zu}$ allererst eine Frage dessen, was uns untereinander und miteinander wichtig ist und nicht, was »der abstrakte Staat « für uns erledigen soll. Derzeit dürften Gesundheits- und Pflegefragen die größte Akzeptanz haben vor der Förderung von Kindern. Strittiger wird es beim Anspruch, jeder möge über Arbeit zu seinem Leben beitragen und wieviel Eigen- und Fremd- die Nationalen bekommen mehr Abschiebungen, die Globalisten bekommen mehr Deutschkurse, der Mittelstand mehr Geld für Facharbeiterintegration usw.

Wird auch die deutsche Kommission für gleiche Lebensverhältnisse enden mit Förderprogrammen für WLAN und Ärzte auf dem Land, Wohnungsmillionen für die Stadt und Entschuldung der Pleitekommunen? Ist dann die Krise der Demokratiegebannt mit Hilfe von Steuermillionen? Erledigt Frankreich seine Krise mit staatlichen Erhöhungen von Renten und Kleinsteinkommen, mit Dezentralisierung und schlanker Verwaltung für den Mittelstand und entschlossenerer Klimapolitik(für die Gebildeten)?

Stellen unsere Demokratien die "gefühlte Gleichheit und Gerechtigkeit« her durch ziel- und milieugruppenspezifische Entscheidungen, die am Ende allen das Gefühl gibt, sie seien gehört und wichtig gewesen? Jürgen Habermas kritisiert dies und nennt es eine "normativ entkernte Politik«, die der Bürgerschaft etwas vorenthält, was diese dazu veranlasst "abzuwandern", sich in vielen "Kleinkriegen « (Stuttgart 21/ Gelbwesten / pro und contra Flüchtlinge) zu verkämpfen. Es sei nicht getan mit dem Verzicht auf große Projekte, Verzicht auf Visionen, Verzicht auf die Versprechungen des Sozialstaates. Immer wieder zeigen große grenzüberschreitende Wellen der Mobilisierung in westlich geprägten Demokratien wie die Klima-Freitags-Bewegung von Greta Thumberg, dass Menschen "große Projekte« wollen, dass sie Visionen brauchen, sich daran orientieren.

Auch in Macrons Bürgerdialogbilanz schimmert eine "alte "Vision auf, die vom 
Aufstieg: Sein wichtigstes Projekt bleibe, die Chancen für den sozialen Aufstieg zu erhöhen (FAZ 27.4.2019). Er wolle die Sezession ganzer Wohngebiete nicht hinnehmen...die republikanische Wiedereroberung der Gebiete habe begonnen. Würde je eine deutsche Kommissionspolitik die "Wiedereroberung ländlicher Räume und städtischer Wohn- und Quartiersprobleme durch »den demokratischen und sozialen Bundesstaat " (Art. 20) verkünden? Welche positive Vision steht hinter den deutschen Wohlfahrtsverbänden, wenn sie publizistisch den Kampf gegen Armut und Hartz IV führen und für Vielfalt werben? Eine bunte Republik, in der keiner Not leidet? Welche Vision steht hinter kommunalen Spitzenverbänden, wenn sie mehr Geld vom Nationalstaat gegen Verschuldungen wollen? Eine lokale selbstverwaltete Demokratie, die den Interventionen von außen trotzen kann?

Wer sozialpolitisch bei den wirklichen, chronischen und schärfsten Ungleichheiten ansetzen will, wird der territorialen Ungleichheit folgend Quartiere vorfinden, in denen alle Problemlagen zusammen und langjährig auftreten. Dort lässt sich präzise aufzeigen, welche $z$. T. anderswo selbstverständliche Leistungen und Verhaltensweisen nicht mehr gelingen oder nur mit immensem Aufwand, von der Sicherheit auf der Straße bis zum Schulerfolg in der Klasse, der Gesundheitsprävention bis zur Schuldnerberatung. Hier wird nur ein integrierter Ansatz gelingen, der über Wohlfahrt hinausdenkt, der öffentliche Hand und intermediäre Träger effizienzorientiert zusammenbindet, der Sozialkapital und Bürgerbeteiligung hineindenkt, der sozial nicht mehr reduziert auf »Sozialpolitik « sondern das Sicherheitswesen, den Städtebau und die Bildungspolitik einbezieht. Unsere derzeitige Sozialpolitik trägt in sich das Paradoxon, dass sie keinen Mangel an Zielen, Methoden und perfekten Standards hat, aber einen Mangel an selbstverantworteten Handlungsräumen mit Eigenverantwortung, Beteiligung und messbaren Effizienzzielen.

Wie sieht ein ganzheitliches Konzept aus, eine »integrierte Stadtentwicklung « wie sie wortreich 2007 in Leipzig formuliert wurde auf einem dt.-franz. Gipfel und nun fortgeschrieben werden soll? Wenn soziale Probleme den Zusammenhalt anfangen zu gefährden, erfolgt in Deutschland der Ruf nach dem Bundesstaat. Egal ob Pflege, Kinderbetreuung, Sozialhilfe oder Digitalisierung, es erfolgt mit immer den gleichen
Folgen. Dem Geld folgt die Verregelung und Nivellierung. Die Kommunen folgen der Zuschusspolitik oder Zuweisungspolitik (z.B. Flüchtlinge) ohne Einwirkungsmöglichkeiten an den Ursachen. Stehen die Kommunen unter großem finanziellen Druck, verkaufen sie ihre Einflussinstrumente (Wohnbaugesellschaften etc.) und stellen ihre Unternehmensteile unter den Kostendruck des Marktes und handeln »für die Armen« Gutscheine aus. So besehen reduziert sich integrierte Stadtentwicklung zu einer Frage nach mehr staatlichem Geld und der Hoffnung, Bund und Länder mögen die Probleme der Quartiere nicht noch verschärfen durch Sparpolitik in Schulen und Polizei. Und wenn es denn sein muss, lassen sich einige Planstellen für Quartiersmanagement bezahlen. Schnell reduziert sich ein Gesamtkonzept zu einem fürsorglichen Betreuungskonzept. Genau diese Gefahr besteht bei fortschrittlich gedachten Sozialraumkonzepten bei der Mobilisierung vieler für die Nöte Einzelner z.B. in der Pflege. Dann geht es ganz am Ende nur darum, dass der Staat das Sozialkapital selbst schleunigst schöpfen will.

Die Wirklichkeit heutiger Demokratie ist in einer inklusiven Gesellschaft angekommen, die es weder vermag noch hinbekommen wird, alle Gesellschaftsmitglieder auf ein Lebensmodell und Versorgungsniveau $\mathrm{zu}$ integrieren, dieses $\mathrm{zu}$ versprechen. Sie muss mit Ungleichheiten und völlig verschiedenen Lebensentwürfen und Ungleichzeitigkeiten klarkommen. Sie darf den elementaren Wunsch nach $\mathrm{Zu}$ sammenhalt und Zusammengehörigkeit, nach gleichen »Rechten und Pflichten" nicht aufgeben- die Menschen wollen diesen »inklusiven « Zusammenhalt, auch wenn jeder für sich »exklusiv«, besonders sein will. Der kleinste gemeinsame Nenner inklusiver Gesellschaften sind deren öffentliche Institutionen, eine funktionstüchtige Verwaltung und ein glaubwürdiger Rechtsstaat, dann die sozialen Einrichtungen und schließlich die ganze Stadtgestalt und Infrastruktur- aber nicht umgekehrt. Wer nur über Ausstattung und Versorgung operieren will, stärkt keine Demokratie mit deren Aufforderung, dass es »die Regierung aller durch alle für alle« ist.

Der zusätzlich hohe Anspruch einer inklusiven Gesellschaft erfordert nicht mehr Mittel zur Gleichheit, sondern mehr Mut und Toleranz zur Ungleichheit, zum Leben unter den verschiedensten Ausgangsbedingungen - allerdings bei gleichen Spielregeln. Und dafür stehen vor allem staatliche Institutionen, eine funktionstüchtige Verwaltung und der Rechtsstaat. Wo in den genannten Quartieren weder Integration gelungen ist - gleich ob die Ursachen weit außerhalb dieser Quartiere liegen - noch Inklusion gelebt wird, weil es eher der Parallelweltenlogik folgt und keine Chancengerechtigkeit für die Kinder-Jugendausbildung besteht, hat sich zu viel angehäuft oder »abgelagert", um noch individuell bewältigt zu werden.

Eine Kommission, die sich der gleichwertigen Lebensverhältnisse annimmt hat die Chance, sich nicht an Wohlstands- oder Niveauunterschieden zu orientieren, sondern an den Einstellungen der Menschen, an Grundrechten und Grundwerten. Ebenso können Wohlfahrtsverbände ihre Bilanz zur Demokratiekrise neu ziehen. Sie haben kritisch zu prüfen, ob sie einfach staatlich alimentiert Dienstleistungen sicherstellen, ob sie mit dem ihnen verbliebenen Sozialkapital noch nachhaltig umgehen, und ob sie an den Grundlagen demokratischer Sozialstaatlichkeit konstruktiv mitwirken, die mehr als bisher die Fragen der Solidarität, Inklusion und des Miteinanders aufrufen.

\section{Literatur}

\section{Berlin-Institut für Bevölkerung und}

Entwicklung \& Wüstenrot Stiftung ( $\mathrm{Hg}$.)

(2019): Teilhabeatlas Deutschland (https:// www.berlin-institut.org/fileadmin/user_ upload/Teilhabeatlas/Teilhabe_Online.pdf)

Faus, Rainer / Storks, Simon (2019): Das pragmatische Einwanderungsland: Was die Deutschen über Migration denken; Bonn: FriedrichEbert-Stiftung. Online abrufbar unter: http:// library.fes.de/pdf-files/fes/15213-20190402.pdf Herlyn, Ulfert (1977): Infrastrukturpolitik als sozialpolitische Aufgabe, in: Ferber, Christian von / Kaufmann, Franz-Xaver (Hg.): Soziologie und Sozialpolitik, Sonderheft der Kölner Zeitschrift für Soziologie und Sozialpsychologie, 19/1977, Opladen: Westdeutscher Verlag, S. 577-590.

Hummel, Konrad (1995): Bürgerengagement; Freiburg: Lambertus Verlag.

Hummel, Konrad (2016): Demokratie in den Städten; Baden-Baden: Nomos.

Hummel, Konrad (2019): Vielfaltsquartiere und Integration, in: BdW 4/2019, S. 129-132.

Van Deth, Jan W. (2014): Demokratie in der Großstadt, der Mannheimer Demokratie Audit; Wiesbaden: Springer.

Wendt, Wolf Rainer (2014): Sorgen für

Wohlfahrt, Baden-Baden: Nomos. 